\title{
The Forgotten Topological Index of Four Operations on Some Special Graphs
}

\author{
Sirous Ghobadi ${ }^{1, a}$,, Mobina Ghorbaninejad ${ }^{2, b}$ \\ 1,2 Department of Mathematics, Qaemshahr Branch, Islamic Azad University, \\ Qaemshahr, Iran. \\ a GhobadiMath46@gmail.com, b Ghorbani325@gmail.com
}

Keywords: F-index, F-sums, Degree, operation on graphs

\begin{abstract}
For a graph, the forgotten topological index (F-index) is defined as the sum of cubes of degrees of vertices. In 2009, Eliasi and Taeri [M. Eliasi, B. Taeri, Four new sums of graphs and their wiener indices, Discrete Appl. Math. 157 (2009) 794-803] introduced four new sums (F-sums) of graphs. In this paper we study the F-index for the F-sums of some special well-known graphs.
\end{abstract}

\section{Introduction}

For a graph $G=(V, E)$ with vertex set $V=V(G)$ and edge set $E=E(G)$, the degree of a vertex $v$ in $G$ is the number of edges incident to $v$ and denoted by $d_{G}(v)$. In chemical graph theory, a topological index is a number related to a graph which is structurally invariant. One of the oldest most popular and extremely studied topological indices are well-known Zagreb indices first introduced in 1972 by Gutman and Trinajestic [6] as follows:

For a graph $G$ with a vertex set $V(G)$ and an edge set $E(G)$, the first and second Zagreb indices are defined as

and

$$
M_{1}(G)=\sum_{v \in V(G)} d_{G}^{2}(v)=\sum_{u v \in E(G)}\left[d_{G}(u)+d_{G}(v)\right]
$$

$$
M_{2}(G)=\sum_{u v \in E(G)} d_{G}(u) d_{G}(v) \text { respectively. }
$$

In [6], beside the first Zagreb index, another topological index defined as

$$
F(G)=\sum_{v \in V(G)} d_{G}^{3}(v)=\sum_{u v \in E(G)}\left[d_{G}^{2}(u)+d_{G}^{2}(v)\right]
$$

However this index, except (implicitly) in a few works about the general first Zagreb index $[9,10]$ and the Zeroth-Order general Randic index [8], was not further studied till then, except in a recent article by Furtula and Gutman [5], where they reinvestigated this index and studied some basic properties of this index. They proposed that $F(G)$ be named the forgotten topological index, or shortly the F-index.

The extremal trees that maximize or minimize the F-index is obtained by Abdo et. al. in [1]. De $N$. et. al. studied behavior of $\mathrm{F}-$ index under several operations and applied their results to find the F-index of different chemically interesting molecular graphs and nano-Structures [3].

In this work we will study the F-index of four operations on Paths, Cycles, Stars and Complete graphs. For this purpose we recall four related graphs as follows:

(a) $S(G)$ is the graph obtained by inserting an additional vertex in each edge of $G$. Equivalently, each edge of $G$ is replaced by a Path of length 2 .

(b) $R(G)$ is obtained from $G$ by adding a new vertex corresponding to each edge of $G$, then joining each new vertex to the end vertices of the corresponding edge.

(c) $Q(G)$ is obtained from $G$ by inserting a new vertex into each edge of $G$, then joining with edges those pairs of new vertices on adjacent edges of $G$. 
(d) $T(G)$ has as its vertices the edges and vertices of G. Adjacency in $T(G)$ is defined as adjacency or incidence for corresponding elements of $G$.

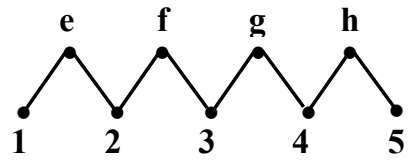

$\mathrm{S}\left(\mathrm{P}_{5}\right)$

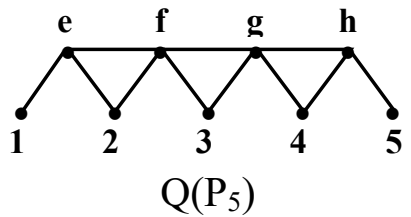

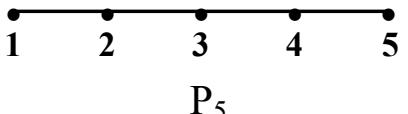

$\mathrm{P}_{5}$

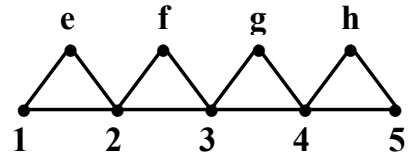

$\mathrm{R}\left(\mathrm{P}_{5}\right)$

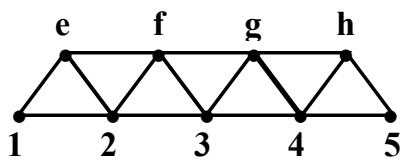

$\mathrm{T}\left(\mathrm{P}_{5}\right)$

Fig1. $\mathrm{P}_{5}, \mathrm{~S}\left(\mathrm{P}_{5}\right), \mathrm{R}\left(\mathrm{P}_{5}\right), \mathrm{Q}\left(\mathrm{P}_{5}\right), \mathrm{T}\left(\mathrm{P}_{5}\right)$

The graph $\mathrm{P}_{5}$
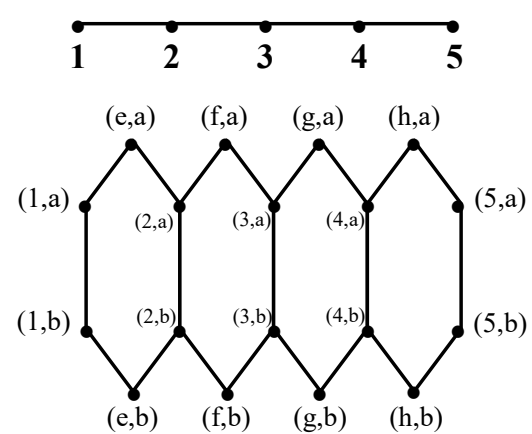

$\mathrm{P}_{5}+{ }_{\mathrm{S}} \mathrm{P}_{2}$

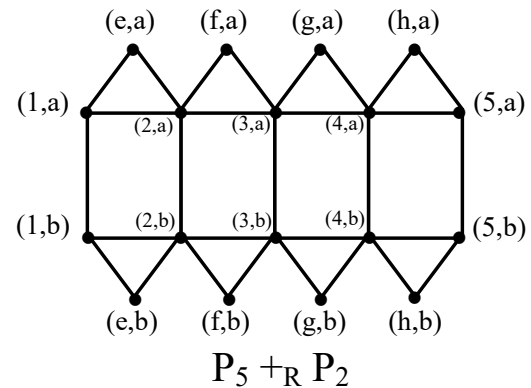

The graph $\mathrm{P}_{2}$
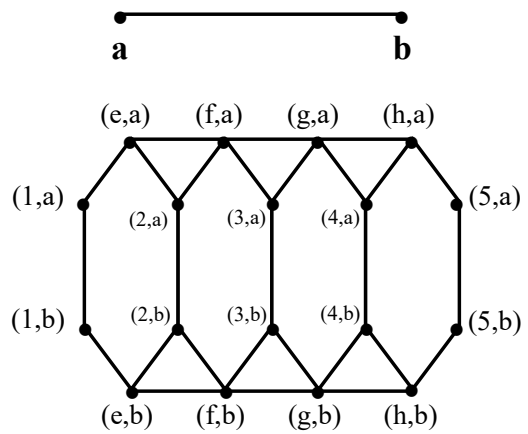

$\mathrm{P}_{5}+\mathrm{Q}_{2}$

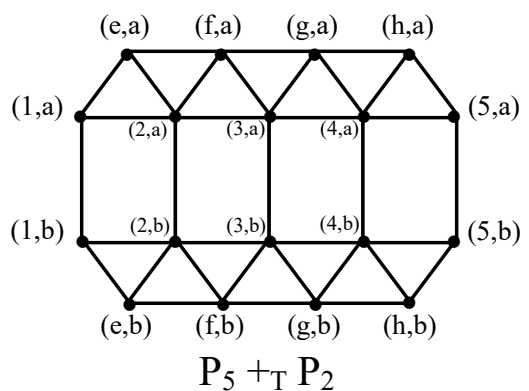

Fig 2. Graphs $\mathrm{P}_{5}+{ }_{F} \mathrm{P}_{2}$

The graph $S(G)$ and $T(G)$ are called the subdivision and total graph of $G$, respectively. For more details on these operations we refer the reader to [2].

If $G$ is $P_{5}$, then $S\left(P_{5}\right), R\left(P_{5}\right), Q\left(P_{5}\right)$ and $T\left(P_{5}\right)$ are shown in Fig 1.

Suppose that $G_{1}$ and $G_{2}$ are two connected graphs. Based on these operations above, Eliasi and Taeri [4] introduced four new operations on these graphs in the following:

Let $F \in\{S, R, Q, T\}$. The F-sum of $G_{1}$ and $G_{2}$, denoted by $G_{1}+{ }_{F} G_{2}$ is a graph with the set of vertices $V\left(G_{1}+_{F} G_{2}\right)=\left(V\left(G_{1}\right) \cup E\left(G_{1}\right)\right) \times V\left(G_{2}\right)$ and two vertices $\left(u_{1}, u_{2}\right)$ and $\left(v_{1}, v_{2}\right)$ of 
$G_{1}+{ }_{F} G_{2}$ are adjacent if and only if $\left[u_{1}=v_{1} \in V\left(G_{1}\right)\right.$ and $\left.u_{2} v_{2} \in E\left(G_{2}\right)\right]$ or $\left[u_{2}=v_{2} \in\right.$ $V\left(G_{2}\right)$ and $\left.u_{1} v_{1} \in E\left(F\left(G_{1}\right)\right)\right]$.

$P_{5}+{ }_{S} P_{2}, P_{5}+{ }_{R} P_{2}, P_{5}+{ }_{Q} P_{2}$ and $P_{5}+{ }_{T} P_{2}$ are shown in Fig 2.

In [4], Eliasi and Taeri obtained the expression for the wiener index $\mathrm{W}\left(G_{1}+{ }_{F} G_{2}\right)$ in terms of $W\left(F\left(G_{1}\right)\right)$ and $W\left(G_{2}\right)$. In [7] Hanyuan Deng et. al. obtained the Zagreb indices of four operations on connected graphs. Here, we will study the F-index for the F-sums of Paths, Cycles, Stars and Complete graphs.

\section{The $\mathbf{F}$-index for $\mathbf{F}$-sums of some special graphs}

In the following four Theorems let $G_{1}$ and $G_{2}$ be two Path of order $n_{1}$ and $n_{2}$ respectively with $\left|G_{1}\right|=e_{1}$ and $\left|G_{2}\right|=e_{2}$. At first we consider the case $F=S$.

\section{Theorem 1.}

$$
F\left(G_{1}+_{S} G_{2}\right)=2 e_{2} M_{1}\left(G_{1}\right)+6 e_{1} M_{1}\left(G_{2}\right)+n_{1} F\left(G_{2}\right)+n_{2} F\left(G_{1}\right)+8 e_{1} n_{2}+16 e_{2} n_{1}-24 e_{2}
$$

Proof. Let $d(u, v)=d_{G_{1}+{ }_{s} G_{2}}(u, v)$ be the degree of vertex $(u, v)$ in the graph $G_{1}+_{s} G_{2}$.

$$
\begin{aligned}
F\left(G_{1}+{ }_{S} G_{2}\right) & =\sum_{\left(u_{1}, v_{1}\right)\left(u_{2}, v_{2}\right) \in E\left(G_{1}+G_{S}\right)}\left[d^{2}\left(u_{1}, v_{1}\right)+d^{2}\left(u_{2}, v_{2}\right)\right] \\
& =\sum_{u \in V\left(G_{1}\right)} \sum_{v_{1} v_{2} \in E\left(G_{2}\right)}\left[d^{2}\left(u, v_{1}\right)+d^{2}\left(u, v_{2}\right)\right] \\
& +\sum_{v \in V\left(G_{2}\right)} \sum_{u_{1} u_{2} \in E\left(S\left(G_{1}\right)\right)}\left[d^{2}\left(u_{1}, v\right)+d^{2}\left(u_{2}, v\right)\right] \\
& =I_{1}+I_{2}
\end{aligned}
$$

Then

$$
\begin{aligned}
I_{1} & =\sum_{u \in V\left(G_{1}\right)} \sum_{v_{1} v_{2} \in E\left(G_{2}\right)}\left[d^{2}\left(u, v_{1}\right)+d^{2}\left(u, v_{2}\right)\right] \\
& =\sum_{u \in V\left(G_{1}\right)} \sum_{v_{1} v_{2} \in E\left(G_{2}\right)}\left[2 d_{G_{1}}^{2}(u)+2 d_{G_{1}}(u)\left(d_{G_{2}}\left(v_{1}\right)+d_{G_{2}}\left(v_{2}\right)\right)+\left(d_{G_{2}}^{2}\left(v_{1}\right)+d_{G_{2}}^{2}\left(v_{2}\right)\right)\right] \\
& =\sum_{u \in V\left(G_{1}\right)}\left[2 e_{2} d_{G_{1}}^{2}(u)+2 d_{G_{1}}(u) M_{1}\left(G_{2}\right)+F\left(G_{2}\right)\right] \\
& =2 e_{2} M_{1}\left(G_{1}\right)+4 e_{1} M_{1}\left(G_{2}\right)+n_{1} F\left(G_{2}\right)
\end{aligned}
$$

and

$$
\begin{aligned}
I_{2} & =\sum_{v \in V\left(G_{2}\right)} \sum_{\substack{u_{1} u_{2} \in E\left(S\left(G_{1}\right)\right) \\
u_{1} \in V\left(G_{1}\right), u_{2} \in V\left(S\left(G_{1}\right)\right)-V\left(G_{1}\right)}}\left[d^{2}\left(u_{1}, v\right)+d^{2}\left(u_{2}, v\right)\right] \\
& =\sum_{v \in V\left(G_{2}\right)} \sum_{\substack{u_{1} u_{2} \in E\left(S\left(G_{1}\right)\right) \\
u_{1} \in V\left(G_{1}\right), u_{2} \in V\left(S\left(G_{1}\right)\right)-V\left(G_{1}\right)}}\left[\left(d_{S\left(G_{1}\right)}\left(u_{1}\right)+d_{G_{2}}(v)\right)^{2}+d_{S\left(G_{1}\right)}^{2}\left(u_{2}\right)\right]
\end{aligned}
$$




$$
\begin{aligned}
& =\sum_{v \in V\left(G_{2}\right)} \sum_{\substack{u_{1} u_{2} \in E\left(S\left(G_{1}\right)\right) \\
u_{1} \in V\left(G_{1}\right), u_{2} \in V\left(S\left(G_{1}\right)\right)-V\left(G_{1}\right)}}\left[\left(d_{S\left(G_{1}\right)}^{2}\left(u_{1}\right)+d_{S\left(G_{1}\right)}^{2}\left(u_{2}\right)\right)+d_{G_{2}}^{2}(v)+2 d_{S\left(G_{1}\right)}\left(u_{1}\right) d_{G_{2}}(v)\right] \\
& =\sum_{v \in V\left(G_{2}\right)}\left[F\left(S\left(G_{1}\right)\right)+2 e_{1} d_{G_{2}}^{2}(v)+2\left(4 n_{1}-6\right) d_{G_{2}}(v)\right] \\
& =n_{2} F\left(S\left(G_{1}\right)\right)+2 e_{1} M_{1}\left(G_{2}\right)+4 e_{2}\left(4 n_{1}-6\right)
\end{aligned}
$$

Hence

$$
F\left(G_{1}+{ }_{S} G_{2}\right)=2 e_{2} M_{1}\left(G_{1}\right)+4 e_{1} M_{1}\left(G_{2}\right)+n_{1} F\left(G_{2}\right)+n_{2} F\left(S\left(G_{1}\right)\right)+2 e_{1} M_{1}\left(G_{2}\right)+4 e_{2}\left(4 n_{1}-6\right)
$$

Note that $F\left(S\left(G_{1}\right)\right)=F\left(G_{1}\right)+8 e_{1}$. We then have the proof.

In the next three Theorems let $X$ and $Y$ be the sets of endvertices of Paths $G_{1}$ and $G_{2}$ respectively. Then $|X|=|Y|=2$ and $d_{G_{1}}(x)=d_{G_{2}}(y)=1$ for $x \in X$ and $y \in Y$.

\section{Theorem 2.}

\section{Proof.}

$$
\begin{aligned}
F\left(G_{1}+{ }_{R} G_{2}\right) & =10 e_{1} M_{1}\left(G_{2}\right)+8 e_{2} M_{1}\left(G_{1}\right)+4\left(e_{2}+1\right) F\left(G_{1}\right)+\left(e_{1}+1\right) F\left(G_{2}\right) \\
& +112 e_{1} e_{2}+36 e_{1}-56 e_{2}-24
\end{aligned}
$$

$$
\begin{aligned}
& F\left(G_{1}+{ }_{R} G_{2}\right)=\sum_{\left(u_{1}, v_{1}\right)\left(u_{2}, v_{2}\right) \in E\left(G_{1}+{ }_{R} G_{2}\right)}\left[d^{2}\left(u_{1}, v_{1}\right)+d^{2}\left(u_{2}, v_{2}\right)\right] \\
& =\sum_{u \in X} \sum_{v_{1} v_{2} \in E\left(G_{2}\right)}\left[d^{2}\left(u, v_{1}\right)+d^{2}\left(u, v_{2}\right)\right] \\
& +\sum_{u \in V\left(G_{1}\right)-X} \sum_{v_{1} v_{2} \in E\left(G_{2}\right)}\left[d^{2}\left(u, v_{1}\right)+d^{2}\left(u, v_{2}\right)\right] \\
& +\sum_{v \in V\left(G_{2}\right)} \sum_{\substack{u_{1} u_{2} \in E\left(R\left(G_{1}\right)\right) \\
u_{1}, u_{2} \in V\left(G_{1}\right)}}\left[d^{2}\left(u_{1}, v\right)+d^{2}\left(u_{2}, v\right)\right] \\
& +\sum_{v \in Y} \sum_{u_{1} u_{2} \in E\left(R\left(G_{1}\right)\right)}\left[d^{2}\left(u_{1}, v\right)+d^{2}\left(u_{2}, v\right)\right] \\
& u_{1} \in V\left(G_{1}\right), u_{2} \in V\left(R\left(G_{1}\right)\right)-V\left(G_{1}\right) \\
& +\sum_{v \in V\left(G_{2}\right)-Y} \sum_{u_{1} u_{2} \in E\left(R\left(G_{1}\right)\right)}\left[d^{2}\left(u_{1}, v\right)+d^{2}\left(u_{2}, v\right)\right] \\
& u_{1} \in V\left(G_{1}\right), u_{2} \in V\left(R\left(G_{1}\right)\right)-V\left(G_{1}\right) \\
& =I_{1}+I_{2}+I_{3}+I_{4}+I_{5} .
\end{aligned}
$$

Then$$
I_{1}=\sum_{u \in X} \sum_{v_{1} v_{2} \in E\left(G_{2}\right)}\left[d^{2}\left(u, v_{1}\right)+d^{2}\left(u, v_{2}\right)\right]
$$ 


$$
\begin{aligned}
& =\sum_{u \in X} \sum_{v_{1} v_{2} \in E\left(G_{2}\right)}\left[8 d_{G_{1}}^{2}(u)+\left(d_{G_{2}}^{2}\left(v_{1}\right)+d_{G_{2}}^{2}\left(v_{2}\right)\right)+4 d_{G_{1}}(u)\left(d_{G_{2}}\left(v_{1}\right)+d_{G_{2}}\left(v_{2}\right)\right)\right] \\
& =\sum_{u \in X}\left[8 e_{2} d_{G_{1}}^{2}(u)+F\left(G_{2}\right)+4 d_{G_{1}}(u) M_{1}\left(G_{2}\right)\right] \\
& =16 e_{2}+2 F\left(G_{2}\right)+8 M_{1}\left(G_{2}\right) .
\end{aligned}
$$

Similar to the case $I_{1}$ we have

$$
I_{2}=\sum_{u \in V\left(G_{1}\right)-X}\left[8 e_{2} d_{G_{1}}^{2}(u)+F\left(G_{2}\right)+4 d_{G_{1}}(u) M_{1}\left(G_{2}\right)\right]
$$

Since for $u \in V\left(G_{1}\right)-X$ there are $n_{1}-2$ vertices of order 2 then $I_{2}=\left(n_{1}-2\right)\left(32 e_{2}+F\left(G_{2}\right)+8 M_{1}\left(G_{2}\right)\right)$.

Now we have

$$
\begin{aligned}
I_{3} & =\sum_{v \in V\left(G_{2}\right)} \sum_{\substack{u_{1} u_{2} \in E\left(R\left(G_{1}\right)\right) \\
u_{1}, u_{2} \in V\left(G_{1}\right)}}\left[d^{2}\left(u_{1}, v\right)+d^{2}\left(u_{2}, v\right)\right] \\
& =\sum_{v \in V\left(G_{2}\right)} \sum_{\substack{u_{1} u_{2} \in E\left(R\left(G_{1}\right)\right) \\
u_{1}, u_{2} \in V\left(G_{1}\right)}}\left[d_{R\left(G_{1}\right)}^{2}\left(u_{1}\right)+d_{R\left(G_{1}\right)}^{2}\left(u_{2}\right)+2 d_{G_{2}}^{2}(v)+2 d_{G_{2}}(v)\left(d_{R\left(G_{1}\right)}\left(u_{1}\right)+d_{R\left(G_{1}\right)}\left(u_{2}\right)\right)\right]
\end{aligned}
$$

Note that $u_{1}, u_{2} \in V\left(G_{1}\right)$ and $u_{1} u_{2} \in E\left(R\left(G_{1}\right)\right)$ iff $u_{1} u_{2} \in E\left(G_{1}\right)$ and $d_{R\left(G_{1}\right)}\left(u_{i}\right)=$ $2 d_{G_{1}}\left(u_{i}\right), \quad i=1,2$. Then

$$
\begin{aligned}
I_{3} & =\sum_{v \in V\left(G_{2}\right)} \sum_{\substack{u_{1} u_{2} \in E\left(G_{1}\right) \\
u_{1}, u_{2} \in V\left(G_{1}\right)}}\left[4\left(d_{G_{1}}^{2}\left(u_{1}\right)+d_{G_{1}}^{2}\left(u_{2}\right)\right)+2 d_{G_{2}}^{2}(v)+4 d_{G_{2}}(v)\left(d_{G_{1}}\left(u_{1}\right)+d_{G_{1}}\left(u_{2}\right)\right)\right] \\
& =\sum_{v \in v\left(G_{2}\right)}\left[4 F\left(G_{1}\right)+2 e_{1} d_{G_{2}}^{2}(v)+4 d_{G_{2}}(v) M_{1}\left(G_{1}\right)\right] \\
& =4 n_{2} F\left(G_{1}\right)+2 e_{1} M_{1}\left(G_{2}\right)+8 e_{2} M_{1}\left(G_{1}\right) . \\
I_{4} & =\sum_{v \in Y}\left[d_{R\left(G_{1}\right)}^{2}\left(u_{1}\right)+d_{R\left(G_{1}\right)}^{2}\left(u_{2}\right)+d_{G_{2}}^{2}(v)+2 d_{R\left(G_{1}\right)}\left(u_{1}\right) d_{G_{2}}(v)\right] \\
u_{1} \in V\left(G_{1}\right), u_{2} \in V\left(R\left(G_{1}\right)\right)-v\left(G_{1}\right) &
\end{aligned}
$$

Since $\quad d_{R\left(G_{1}\right)}(u)=\left\{\begin{array}{lll}2 & \text { if } & u \in X \\ 4 & \text { if } & u \in V\left(G_{1}\right)-X\end{array} \quad\right.$ then

$\sum_{\substack{u_{1} u_{2} \in E\left(R\left(G_{1}\right)\right) \\ \text { and }}} d_{R\left(G_{1}\right)}\left(u_{1}\right)=2+4+\cdots+4+2=2+\left(2 n_{1}-4\right) \cdot 4+2=8 n_{1}-12$

$\sum_{u_{1} u_{2} \in E\left(R\left(G_{1}\right)\right)} d_{R\left(G_{1}\right)}^{2}\left(u_{1}\right)=32 n_{1}-56$

then

$I_{4}=\sum_{v \in Y}\left[\left(32 n_{1}-56\right)+8 e_{1}+2 e_{1} d_{G_{2}}^{2}(v)+2\left(8 n_{1}-12\right) d_{G_{2}}(v)\right]$ 


$$
\begin{aligned}
& =2\left(32 n_{1}-56\right)+16 e_{1}+4 e_{1}+4\left(8 n_{1}-12\right) \\
& =96 n_{1}+20 e_{1}-160
\end{aligned}
$$

Similar to the case $I_{4}$, we have

$$
\begin{aligned}
I_{5} & =\sum_{v \in V\left(G_{2}\right)-Y}\left[\left(32 n_{1}-56\right)+8 e_{1}+2 e_{1} d_{G_{2}}^{2}(v)+2\left(8 n_{1}-12\right) d_{G_{2}}(v)\right] \\
& =\left(n_{2}-2\right)\left[\left(32 n_{1}-56\right)+8 e_{1}+8 e_{1}+4\left(8 n_{1}-12\right)\right] \\
& =\left(n_{2}-2\right)\left(64 n_{1}+16 e_{1}-104\right) .
\end{aligned}
$$

Hence the proof.

\section{Theorem 3.}

\section{Proof.}

$$
\begin{aligned}
F\left(G_{1}+{ }_{Q} G_{2}\right) & =\left(e_{1}+1\right) F\left(G_{2}\right)+\left(e_{2}+1\right)\left(M_{1}\left(G_{1}\right)+2 M_{2}\left(G_{1}\right)\right) \\
& +108 e_{1} e_{2}-82 e_{2}+48 e_{1}-70
\end{aligned}
$$

$$
\begin{aligned}
& F\left(G_{1}+{ }_{Q} G_{2}\right)=\sum_{\left(u_{1}, v_{1}\right)\left(u_{2}, v_{2}\right) \in E\left(G_{1}+{ }_{Q} G_{2}\right)}\left[d^{2}\left(u_{1}, v_{1}\right)+d^{2}\left(u_{2}, v_{2}\right)\right] \\
& =\sum_{u \in V\left(G_{1}\right)} \sum_{v_{1} v_{2} \in E\left(G_{2}\right)}\left[d^{2}\left(u, v_{1}\right)+d^{2}\left(u, v_{2}\right)\right] \\
& +\sum_{v \in Y} \sum_{u_{1} u_{2} \in E\left(Q\left(G_{1}\right)\right)}\left[d^{2}\left(u_{1}, v\right)+d^{2}\left(u_{2}, v\right)\right] \\
& u_{1} \in V\left(G_{1}\right), u_{2} \in V\left(Q\left(G_{1}\right)\right)-V\left(G_{1}\right) \\
& +\sum_{v \in V\left(G_{2}\right)-Y} \sum_{\substack{u_{1} u_{2} \in E\left(Q\left(G_{1}\right)\right) \\
u_{1} \in V\left(G_{1}\right), u_{2} \in V\left(Q\left(G_{1}\right)\right)-V\left(G_{1}\right)}}\left[d^{2}\left(u_{1}, v\right)+d^{2}\left(u_{2}, v\right)\right] \\
& +\sum_{v \in V\left(G_{2}\right)} \sum_{\substack{u_{1} u_{2} \in E\left(Q\left(G_{1}\right)\right) \\
u_{1}, u_{2} \in V\left(Q\left(G_{1}\right)\right)-V\left(G_{1}\right)}}\left[d^{2}\left(u_{1}, v\right)+d^{2}\left(u_{2}, v\right)\right]=I_{1}+I_{2}+I_{3}+I_{4}
\end{aligned}
$$

With respect to the above Theorems its easy to see that

and

$$
I_{1}=24 e_{1} e_{2}-4 e_{2}-8 e_{1}+n_{1} F\left(G_{2}\right)
$$

$$
I_{4}=n_{2} M_{1}\left(G_{1}\right)+2 n_{2} M_{2}\left(G_{1}\right)+20 n_{1} n_{2}-56 n_{2}
$$

For the case $I_{2}$ and $I_{3}$ we have

$$
\begin{aligned}
& I_{2}=\sum_{v \in Y} \sum_{\substack{u_{1} u_{2} \in E\left(Q\left(G_{1}\right)\right) \\
u_{1} \in V\left(G_{1}\right), u_{2} \in V\left(Q\left(G_{1}\right)\right)-V\left(G_{1}\right)}}\left[d_{Q\left(G_{1}\right)}^{2}\left(u_{1}\right)+d_{Q\left(G_{1}\right)}^{2}\left(u_{2}\right)+d_{G_{2}}^{2}(v)+2 d_{G_{1}}\left(u_{1}\right) d_{G_{2}}(v)\right] \\
&=2\left(8 n_{1}-14\right)+2\left(32 n_{1}-60\right)+4 e_{1}+4\left(4 n_{1}-6\right) \\
&=96 n_{1}+4 e_{1}-172 \\
& \text { and } I_{3}=56 n_{1} n_{2}-98 n_{2}-112 n_{1}+8 e_{1} n_{2}-16 e_{1}+196 .
\end{aligned}
$$

Note that $n_{1}=e_{1}+1$ and $n_{2}=e_{2}+1$ we then have 


$$
\begin{aligned}
F\left(G_{1}+{ }_{Q} G_{2}\right) & =I_{1}+I_{2}+I_{3}+I_{4} \\
& =\left(e_{1}+1\right) F\left(G_{2}\right)+\left(e_{2}+1\right)\left(M_{1}\left(G_{1}\right)+2 M_{2}\left(G_{1}\right)\right) \\
& +108 e_{1} e_{2}-82 e_{2}+48 e_{1}-70 .
\end{aligned}
$$

Since $d_{G_{1}+{ }_{T} G_{2}}(u, v)=\left\{\begin{array}{c}d_{G_{1}+{ }_{R} G_{2}}(u, v) \text { for } u \in V\left(G_{1}\right) \text { and } v \in V\left(G_{2}\right) \\ d_{G_{1}+Q G_{2}}(u, v) \text { for } u \in V\left(T\left(G_{1}\right)\right)-V\left(G_{1}\right) \text { and } v \in V\left(G_{2}\right)\end{array}\right.$

we can get the following Theorem by the proofs of Theorems 2 and 3 .

\section{Theorem 4.}

$$
\begin{aligned}
F\left(G_{1}+{ }_{T} G_{2}\right) & =n_{1} F\left(G_{2}\right)+4 n_{2} F\left(G_{1}\right)+M_{1}\left(G_{1}\right)\left(n_{2}+8 e_{2}\right)+2 M_{1}\left(G_{2}\right)\left(e_{1}+4 n_{1}-4\right) \\
& +2 n_{2} M_{2}\left(G_{1}\right)+32 n_{1} e_{2}+8 n_{2} e_{1}+100 n_{1} n_{2}-196 n_{2}-12 e_{1}-48 e_{2}
\end{aligned}
$$

Applying the above four Theorems we have the following Theorems.

Theorem 5. For $n \geq 3$ and $m \geq 2$,
(a) $F\left(C_{n}+{ }_{S} P_{m}\right)=n(72 m-74)$
(b) $F\left(C_{n}+{ }_{R} P_{m}\right)=n(224 m-182)$
(c) $F\left(C_{n}+{ }_{Q} P_{m}\right)=n(128 m-74)$
(d) $F\left(C_{n}+{ }_{T} P_{m}\right)=n(280 m-182)$

Theorem 6. For $n \geq 4$ and $m \geq 2$
(a) $F\left(K_{1, n-1}+{ }_{S} P_{m}\right)=m\left(n^{3}+3 n^{2}+38 n-34\right)-2\left(3 n^{2}+22 n-18\right)$
(b) $F\left(K_{1, n-1}+{ }_{R} P_{m}\right)=8 m\left(n^{3}+9 n-9\right)-2\left(12 n^{2}+31 n-36\right)$
(c) $F\left(K_{1, n-1}+{ }_{Q} P_{m}\right)=m\left(n^{4}+3 n^{2}+30 n-26\right)-2\left(3 n^{2}+22 n-18\right)$
(d) $F\left(K_{1, n-1}+_{T} P_{m}\right)=m\left(n^{4}+7 n^{3}+64 n-64\right)-2\left(12 n^{2}+31 n-36\right)$

Theorem 7. For $n \geq 3$ and $m \geq 2$,
(a) $F\left(K_{n}+{ }_{s} P_{m}\right)=2 n^{4}+4 m n(n-1)+n(n+1)^{3}(m-2)$
(b) $F\left(K_{n}+{ }_{R} P_{m}\right)=2 n(2 n-1)^{3}+4 m n(n-1)+8 n^{4}(m-2)$
(c) $F\left(K_{n}+_{Q} P_{m}\right)=2 n^{4}+4 m n(n-1)^{4}+n(n+1)^{3}(m-2)$
(d) $F\left(K_{n}+_{T} P_{m}\right)=\frac{1}{2} m n(n-1)(2 n-2)^{3}+2 n(2 n-1)^{3}+8 n^{4}(m-2)$

\section{References}

[1] H. Abdo, D. Dimitrov and I. Gutman, On extremal trees with respect to the F-index, CORR abs/ 1509.03574 (2015).

[2] D. M. Cvetkocic, M. Doob, H. Sachs, Spectra of graphs theory and application, Academic press, New York, 1980.

[3] N. De, SM. Nayeem, A. Pal, F-index of some graph operations. Discrete Math. Algorithm Appl. doi: 10.1142/5179383091650057 (2016).

[4] M. Eliasi and B. Taeri, Four new sums of graphs and their wiener indices, Discrete Appl. Math. 157 (2009) 794-803.

[5] B. Furtula, I. Gutman, A forgotten topological index, J. Math. Chem. 53 (2015) 1184-1190.

[6] I. Gutman, N. Trinajstic, Graph theory and molecular orbitals total $\pi$-electron energy of alternant hydrocarbons, Chem. Phys. Let. 17 (1972) 535-538.

[7] Hanyuan Deng, D. Sarala, S. K. Ayyaswamy, S. Balachandran, The Zagreb indices of four operations on graphs, Appl. Math. and Computation. 275 (2016) 422-431.

[8] Y. Hu, X. Li, Y. Shi, T. Xu, I. Gutman, On molecular graphs with Smallest and greatest zerothorder general Randic index, MATCH Commun. Math. Comput. Chem. 54 (2005) 425-434.

[9] X. Li, J. Zheng, A unified approach to the entremal trees for different indices, MATCH Commun. Math. Comput. Chem. 54 (2005) 195-208.

[10] X. Li, H. Zhao, Trees with the first three smallest and largest generalized topological indices, MATCH Commun. Math. Comput. Chem. 50 (2004) 57-62. 